\title{
Multimodal In Vivo Imaging of Tumorigenesis and Response to Chemotherapy in a Transgenic Mouse Model of Mammary Cancer
}

\author{
Jean-Louis Alberini, ${ }^{1,2,3}$ Raphaël Boisgard, ${ }^{1}$ Stéphanie Guillermet, ${ }^{1}$ Karine Siquier, ${ }^{1}$ \\ Benoît Jego, ${ }^{1}$ Benoît Thézé, ${ }^{1}$ Saik Urien, ${ }^{4}$ Keyvan Rezaï, ${ }^{4}$ Emmanuelle Menet, ${ }^{5}$ \\ Renaud Maroy, ${ }^{1}$ Frédéric Dollé, ${ }^{1}$ Bertrand Kühnast, ${ }^{1}$ Bertrand Tavitian ${ }^{6,7}$ \\ ${ }^{1}$ CEA, DSV, I2BM, Service Hospitalier Frédéric Joliot, Laboratoire d'Imagerie Moléculaire Expérimentale, Orsay, France \\ ${ }^{2}$ Service de Médecine nucléaire, Institut Curie, Hôpital René Huguenin, Saint-Cloud, France \\ ${ }^{3}$ Faculté de Médecine, Université Versailles Saint-Quentin, Versailles, France \\ ${ }^{4}$ Service de pharmacologie, Institut Curie, Hôpital René Huguenin, Saint-Cloud, France \\ ${ }^{5}$ Service de pathologie, Institut Curie, Hôpital René Huguenin, Saint-Cloud, France \\ ${ }^{6}$ Université Paris Descartes Sorbonne Paris Cité, Assistance Publique-Hôpitaux de Paris, Hôpital Européen Georges Pompidou, Radiology \\ Department, Paris, France \\ ${ }^{7}$ Université Paris Descartes Sorbonne Paris Cité, INSERM UMR 970, Cardiovascular Research Center - PARCC, 56 rue Leblanc, 75015 , \\ Paris, France
}

\begin{abstract}
Purpose: Transgenic mice expressing the polyoma middle $\mathrm{T}$ oncoprotein (PyMT) in the mammary epithelium were explored by multimodal imaging to monitor longitudinally spontaneous tumor growth and response to chemotherapy.

Procedures: Positron emission tomography (PET) with 2-deoxy-2-[ $\left.{ }^{18} \mathrm{~F}\right]$ fluoro-D-glucose $\left(\left[{ }^{18} \mathrm{~F}\right] \mathrm{FDG}\right)$ and $3^{1}-$ deoxy- $3^{1}-\left[{ }^{18} \mathrm{~F}\right]$ fluorothymidine $\left(\left[{ }^{18} \mathrm{~F}\right] \mathrm{FLT}\right)$, single photon emission tomography (SPECT) with [ $\left.\left.{ }^{99 m} \mathrm{Tc}_{\mathrm{T}}\right] \mathrm{TO}_{4}\left({ }^{99 \mathrm{~m}} \mathrm{Tc}\right] \mathrm{TEC}\right), \mathrm{X}$-ray computed tomography, and fluorescent confocal endomicroscopy (FCE) images were acquired during tumor progression in female PyMT mice. Imaging with $\left[{ }^{18} \mathrm{~F}\right] \mathrm{FDG}$ and $\left[{ }^{99 \mathrm{~m}} \mathrm{TC}\right] \mathrm{TEC}$ was also performed in untreated, doxorubicin-treated, and docetaxel-treated PyMT mice. Total tumor volumes were quantified. Tumors were collected and macroscopic and histological examinations were performed.

Results: All PyMT mice developed multifocal tumors of the mammary epithelium that became palpable at 8 weeks of age (W8). Computed tomography (CT) detected tumors at W14, while a clear tumoral uptake of $\left.{ }^{99 m} \mathrm{TC}\right]$ TEC and $\left[{ }^{18} \mathrm{~F}\right] \mathrm{FDG}$ was present as early as W6 and W8, respectively. No contrast between mammary tumors and surrounding tissue was observed at any stage with $\left[{ }^{18} \mathrm{~F}\right] \mathrm{FLT}$. FCE detected an angiogenic switch at W10. Lung metastases were not clearly evidenced by imaging. Doxorubicin and docetaxel treatments delayed tumor growth, as shown by $\left[{ }^{18} \mathrm{~F}\right] \mathrm{FDG}$ and $\left[{ }^{99 \mathrm{~m}} \mathrm{Tc}\right] \mathrm{TEC}$, but tumor growth resumed upon treatment discontinuation. Tumor growth fitted an exponential model with time constant rates of $0.315,0.145$, and
\end{abstract}

Jean-Louis Alberini and Raphaël Boisgard contributed equally to this work. Electronic supplementary material The online version of this article (doi:10.1007/s11307-015-0916-7) contains supplementary material, which is available to authorized users.

Correspondence to: Bertrand Tavitian; e-mail: bertrand.tavitian@inserm.fr 
0.212 week $^{-1}$ in untreated, doxorubicin, and docetaxel groups, respectively. Conclusions: Molecular imaging of mammary tumors in PyMT is precocious, precise, and predictive. $\left[{ }^{18} \mathrm{~F}\right] \mathrm{FDG}-\mathrm{PET}$ and $\left[{ }^{99 \mathrm{~m}} \mathrm{TC}\right] \mathrm{TEC}$ SPECT monitor tumor response to chemotherapy.

Key words: Molecular imaging, Biomarker, Breast cancer, Response to treatment, Transgenic model, Sodium-iodide symporter

\section{Introduction}

The ability of in vivo imaging to recognize tumors in a precocious, precise, and predictive manner improves detection, staging, and response to treatment of cancer. Animal models of cancer can be useful to test imaging methods for tumor progression and response to treatment in longitudinal studies. However, physiological differences between species in drug metabolism, circulation, plasmatic concentration of metabolites and nutriments, etc. may influence imaging results and may reduce the clinical interest of translational studies, especially in models where tumors grow in a xenogeneic environment and/or heterotopically. Transgenic models have the strengths that tumors develop in situ in the native tissue, although tumorigenesis is often a slow and stochastic process, which may render difficult and tedious the constitution of homogeneous groups of animals for comparison purposes.

The transgenic mouse model of mammary carcinoma caused by expression of the polyoma middle $\mathrm{T}$ oncoprotein (PyMT) in the mammary epithelium [1] mimics the Luminal B type of human breast cancer. Although it does not recapitulate all of the human disease (absence of bone and brain metastases, loss of hormone receptors during progression), it offers several advantages for imaging studies of breast carcinogenesis: (i) tumors develop rapidly (23 months) in the mammary glands localized along parasagittal lines in the neck, chest, abdomen, and pelvis parts, (ii) penetrance of the phenotype is $\sim 100 \%$ in heterozygous $\mathrm{PyMT}^{+/-}$female mice, (iii) progression to malignancy offers similarities with that observed during human ductal carcinoma carcinogenesis [2], including frequent occurrence of lymph node and lung metastases at late stages [2], and (iv) PyMT tumors express many of the genes overexpressed in human luminal tumors, including estrogen and progesterone receptors (ER and PR positive) and the sodium-iodide symporter (NIS) [3]. In the present study, we applied in vivo imaging techniques for the assessment of early tumor response in female PyMT mice. We performed X-ray computed tomography (CT), positron emission tomography (PET) with 2-deoxy-2-[ ${ }^{18}$ F $]$ fluoro-D-glucose $\left(\left[{ }^{18} \mathrm{~F}\right] \mathrm{FDG}\right)$ and 3 'deoxy- 3 '- $\left[{ }^{18} \mathrm{~F}\right]$ fluorothymidine $\left(\left[{ }^{18} \mathrm{~F}\right] \mathrm{FLT}\right)$, single photon emission computed tomography (SPECT) with $\left[{ }^{99 \mathrm{~m}} \mathrm{Tc}\right] \mathrm{TcO}_{4}\left(\left[{ }^{99 \mathrm{~m}} \mathrm{Tc}\right] \mathrm{TEC}\right)$, and fluorescent confocal endomicroscopy (FCE) of microvessels in longitudinal weekly follow-up to detect mammary tumors and lung metastases [1]. In vivo imaging was repeated in groups of animals receiving standard chemotherapy for breast cancer: doxorubicin, an anthracyclin, or docetaxel, a taxane. In vivo imaging data were correlated with immunohistochemistry of Ki-67 and NIS expression.

\section{Material and Methods}

\section{Animals}

Animal experiments were approved by the local ethical committee and followed the ARRIVE guidelines of the National Centre for the Replacement, Refinement, and Reduction of Animals in Research (London, UK). Female heterozygous $\mathrm{PyMT}^{-/+}$mice were obtained by crossing wild-type FVB females with heterozygous $\mathrm{PyMT}^{-/+}$ males. Offsprings were genotyped by qualitative PCR. ADN was extracted from $25 \mathrm{~mm}^{3}$ of mouse tail using $100 \mu \mathrm{L}$ of Extract-N$\mathrm{Amp}^{\mathrm{TM}}$ PCR ReadyMix ${ }^{\mathrm{TM}}$ incubated for $10 \mathrm{~min}$ at $25^{\circ} \mathrm{C}$ followed by 3 min at $95^{\circ} \mathrm{C}$. Primers were PyMT3/PyMT and PO up/PO low for transgenic and $\mathrm{FVB}$, respectively.

Animals were housed separately at constant temperature $\left(21^{\circ} \mathrm{C}\right)$ and relative humidity $(60 \%)$ under a regular light/dark schedule. Food and water were freely available. Mice were randomly assigned to the untreated or treated group. Mean total tumor volume (TTV) per mouse was defined as the sum of all tumors visible in the five pairs of mammary glands in a given mouse. Imaging was performed weekly in non-fasted mice anesthetized with $2 \%$ isoflurane. A heat lamp was used to maintain body temperature. Animals were sacrificed under terminal anesthesia at 14 weeks of age (W14) in the untreated group and at W18 in the treated groups. In total, 96 animals were used for imaging and biological experimentations.

\section{Chemotherapy}

Preliminary Toxicity Studies In order to determine the maximal tolerated dose (MTD), the cytotoxic drugs doxorubicin and docetaxel were administered to two groups of five FVB mice. Doxorubicin (Doxorubicin ${ }^{\circledR}$, Teva Pharmaceuticals, France) diluted in $0.9 \% \mathrm{NaCl}$ was injected intraperitoneally (IP) in five mice at a dose of $10 \mathrm{mg} / \mathrm{kg}$ weekly during three successive weeks [4]. Docetaxel (Taxotere ${ }^{\circledR}$, Sanofi-Aventis, France) was diluted in its specific excipient and given to two different groups of four mice either intravenously (IV) or IP at different doses $(15,20$, and $30 \mathrm{mg} / \mathrm{kg}$ ) in three successive administrations at 4-day intervals. After drug administration, all animals were monitored during 2 months by clinical examination, weight assessment every other day, and dental examination every week.

In the doxorubicin-treated group treated with a $10-\mathrm{mg} / \mathrm{kg}$ dose, a $20 \%$ weight loss with a 15-day nadir was observed. No other 
signs of toxicity were observed, and this dose was deemed acceptable. In the group treated with $30 \mathrm{mg} / \mathrm{kg}$ docetaxel IV, a $20 \%$ weight loss was observed, while in the group treated by $30 \mathrm{mg} / \mathrm{kg}$ docetaxel IP, weight loss was $10 \%$ with a 12-day nadir. However, in the IV-treated group, in two cases, we observed severe muscular paralysis leading to muscular atrophy 2 weeks after the first injection, followed by partial recovery at 9 weeks. This side effect was not observed in the group treated by the IP route. Considering the possibility of supplementary toxicity due to anesthesia and in order to perform a longitudinal follow-up, in the present study, docetaxel was administered IP at $20 \mathrm{mg} / \mathrm{kg}$.

\section{Chemotherapy Protocol for Imaging}

Homozygous PyMT mice from the same littermate were randomly assigned to one of the following groups: (i) sham-treated; (ii) three IP injections of doxorubicin, $10 \mathrm{mg} / \mathrm{kg}$ weekly from W9 to W11; and (iii) three IP injections of docetaxel, $20 \mathrm{mg} / \mathrm{kg}$ every 4 days from W9 to W10. These regimens improve chemosensitivity in mouse models of breast cancer ([4] and E. Marangoni and M.F. Poupon, personal communication).

Four mice from each group were imaged with PET from W8 to W18, and four mice from each group were imaged with SPECT from W8 to W18. The administration of cytotoxic drugs was always performed after the SPECT and PET imaging sessions. Body weights were recorded two to three times per week. No tooth loss was observed during the follow-up.

\section{Imaging}

CT Whole body CT scans were acquired on the Fast micro-CT scanner SkyScan 1178 (Skyscan, Kontich, Belgium) operated at $50 \mathrm{keV}$ and a rotational angle of $180^{\circ}$ with averaging of 4 and respiratory gating. Pixel size was $160 \mu \mathrm{m}$, and the total scan duration was approximately $4 \mathrm{~min}$. No contrast medium was administered.

\section{$\left[{ }^{18}\right.$ F]FDG-PET Imaging}

Image Acquisition It has been reported that isoflurane anesthesia induces large variations of glycemia in fasted mice but is not a major confounding factor for reproducibility of $\left[{ }^{18} \mathrm{~F}\right] \mathrm{FDG}-\mathrm{PET}$ [5]. Therefore, in the present study, $\left[{ }^{18} \mathrm{~F}\right] \mathrm{FDG}$ was administered in nonfasted animals and scanning protocols (time after injection and duration) were standardized as recommended.

A first group of PyMT mice $(n=4)$ was imaged weekly from W8 to W14 (except for 1 mouse that was also imaged until W16) after tail vein injection of $7 \mathrm{MBq}(200 \mu \mathrm{Ci})$ of $\left[{ }^{18} \mathrm{~F}\right] \mathrm{FDG}$ (Cisbio, Orsay, France) diluted in $100 \mu \mathrm{l}$ of a saline solution. Under isoflurane anesthesia, serial dynamic PET images $(60 \mathrm{~s} \times 5,120 \mathrm{~s} \times 5,300 \mathrm{~s} \times 3$, $600 \mathrm{~s} \times 3,900 \mathrm{~s} \times 2$ ) were acquired in a microPET Focus 220 (CTIConcorde Microsystems Inc., USA) in 3D mode during $90 \mathrm{~min}$, starting immediately after the injection of $\left[{ }^{18} \mathrm{~F}\right] \mathrm{FDG}$. Time-activity curves showed that $\left[{ }^{18} \mathrm{~F}\right] \mathrm{FDG}$ uptake increased exponentially and reached a plateau approximately 50-60 min after injection.
Image Reconstruction and Analysis Images were reconstructed using OSEM 2D with four iterations and 16 subsets and with a correction attenuation map calculated from the first images [6]. Dynamic images were analyzed with Anatomist software (SHFJ/ CEA, France) using semi-automatic segmentation software (local mean analysis method or LMA) [7]. The metabolic tumor volume (MTV) was assessed as the summed volume of all tumoral mammary glands showing higher $\left[{ }^{18} \mathrm{~F}\right] \mathrm{FDG}$ uptake than the surrounding tissue.

\section{$\left[^{18}\right.$ F]FLT-PET Imaging}

$\left[{ }^{18}\right.$ F]FLT Synthesis $\left[{ }^{18} \mathrm{~F}\right] \mathrm{FLT}$ was synthesized according to a procedure slightly modified from Yun et al. [8] and Oh et al. [9]. Briefly, fluorine- 18 , as dried $\mathrm{K}\left[{ }^{18} \mathrm{~F}\right] \mathrm{F}-\mathrm{K} 222$ complex, was introduced into the precursor 3- $\mathrm{N}$-Boc-5'-O-dimethoxytrityl-3'- $\mathrm{O}$-nosylthymidine (ABX, Germany), in $800 \mu \mathrm{L}$ of acetonitrile at $165^{\circ} \mathrm{C}$ for 1 min. After a rapid purification on a silica cartridge, the $O$ dimethoxytrityl and $N$-Boc protective groups were removed using aqueous $\mathrm{HCl} 4 \mathrm{~N}$ at $90{ }^{\circ} \mathrm{C}$ for $5 \mathrm{~min}$. The final purification was performed on preparative reverse-phase HPLC, eluted with a mixture of aqueous $0.9 \% \mathrm{NaCl}$ and ethanol $(92: 8, v / v)$. $\left[{ }^{18} \mathrm{~F}\right] \mathrm{FLT}$ with chemical and radiochemical purities higher than 95 and $98 \%$, respectively, was obtained in a 15 to $20 \%$ radiochemical yield (decay corrected) with a specific radioactivity ranging from 260 to $330 \mathrm{GBq} / \mu \mathrm{mol}$ (decay corrected).

Image Acquisition Dynamic PET acquisitions were acquired during $90 \mathrm{~min}(60 \mathrm{~s} \times 5,120 \mathrm{~s} \times 5,300 \mathrm{~s} \times 3,600 \mathrm{~s} \times 3,900 \mathrm{~s} \times 2$ images) in four PyMT mice with large tumors (W14) after tail vein injection of $7 \mathrm{MBq}(200 \mu \mathrm{Ci})$ of $\left[{ }^{18} \mathrm{~F}\right] \mathrm{FLT}$ (CEA, Orsay, France) diluted in $100 \mu \mathrm{l}$ of saline solution.

\section{SPECT Imaging with $\left.P^{99 m} T c\right] T E C$}

Image Acquisition SPECT images were performed on a one-head 10 -cm field-of-view parallel-hole collimator gamma camera combined with a CT scanner (Gamma Imager, Biospace Lab, France), $15 \mathrm{~min}$ after tail vein injection of $7 \mathrm{MBq}(200 \mu \mathrm{Ci})$ of $\left[{ }^{99 \mathrm{~m}} \mathrm{Tc}\right] \mathrm{TEC}$ (Cisbio, Orsay, France) diluted in $100 \mu \mathrm{L}$ of saline solution.

Image Analysis Images were reconstructed using an OSEM 2D protocol and analyzed with Amira TM software (FEI, USA). Mammary tumors in PyMT mice showed a clear higher $\left[{ }^{99 \mathrm{~m}} \mathrm{Tc}\right]$ TEC uptake than adjacent tissue. They were manually delineated, and their volume was measured and summed to yield TTV.

\section{In Vivo Fluorescence Imaging}

In vivo fluorescence imaging was performed $15 \mathrm{~min}$ after intravenous injection of $200 \mu \mathrm{l}$ Dextran-FITC (30 mg/ml), using a fibered FCE (Cellvizio ${ }^{\circledR}$ Mauna Kea Technologies, France). The tip of the imaging probe was placed in contact with the abdominal mammary glands. FVB $(n=5)$ and untreated $(n=5)$ and docetaxeltreated $(10 \mathrm{mg} / \mathrm{kg}$ weekly from W9 to W11, $n=5)$ PyMT mice were imaged. Parameters such as total vessel length, vascular density, 
diameter, and morphology of blood vessels were obtained by image analysis using ImageCell ${ }^{\mathrm{TM}}$ software (Mauna Kea Technologies) and automatic segmentation [10].

\section{Ex Vivo Analysis}

Whole Mount In order to assess the tumor diffusion and detect the lymph node involvement, mammary glands were analyzed using the whole mount method. They were defatted, dehydrated, and stained with hematoxylin following the Rosen Laboratory Mammary Gland Whole Mount Protocol [11].

Histology Ki-67 and NIS staining were assessed on formalin-fixed paraffin-embedded tissues of normal and tumoral mammary glands, respectively, in FVB and PyMT mice, at W4, W6, W8, W10, W11, $\mathrm{W} 12$, and $\mathrm{W} 14$ ( $n=2$ for each time point) and in the treated groups. Mammary tumors were excised, fixed in $10 \%$ paraformaldehyde in phosphate-buffered saline (PBS, pH 7.4), dehydrated, and embedded in paraffin. Serial sections were cut at $5 \mu \mathrm{m}$ and stained with hematoxylin-eosin or processed for immunochemistry.

Immunohistochemistry Paraffin-embedded sections were deparaffinized and rehydrated through graded alcohols. Endogenous peroxidases were blocked with $3 \% \mathrm{H}_{2} \mathrm{O}_{2}$ during 5 min at $25{ }^{\circ} \mathrm{C}$ in Tris-buffered solution, washed, and processed for antigen retrieval with PBS (10 mM, pH 6) in a microwave oven (20 min, $750 \mathrm{~W})$.

For Ki-67, sections were incubated with a goat anti-Ki-67 polyclonal IgG (Sc-7846, Santa Cruz Biotechnology Inc., Santa Cruz, USA) as primary antibody and a horse anti-goat IgG (PI9500 , AbCys) as secondary antibody, both at a concentration of $1: 300$ in $0.5 \%$ Tween-PBS. For NIS, sections were incubated with a polyclonal rabbit anti-NIS at a concentration of 1:200. To reduce non-specific interactions of secondary antibodies, tissues were first incubated with $5 \%$ normal serum from the host before incubation at $25{ }^{\circ} \mathrm{C}$ for $1 \mathrm{~h}$ with the primary antibodies. Endogenous peroxidases were blocked with $3 \% \mathrm{H}_{2} \mathrm{O}_{2}$ in water, and immunocomplexes were visualized by the $\mathrm{ABC}$ peroxidase method after staining with DAB for 2 min (Sigma-Aldrich, USA) and counterstained with hematoxylin-eosin. All histological slides were analyzed by an experienced pathologist (EM). In selected areas of hyperplasia, adenoma, and carcinoma localized in mammary glands using HES staining, the Ki-67 index was measured by eye-counting on $500-1,000$ cells.
Tumor Growth Models Modelling of tumor growth was performed with Monolix 3.1 Release 2 (http://software.monolix.org/), a non-linear mixed effect model program that estimates both the structural model parameters as well as the between-subject (mice) and residual variability [12].

Statistical Analysis A nonparametric Mann-Whitney test was used for comparisons of MTV between groups: treated vs. untreated and doxorubicin- $v s$. docetaxel-treated. $P$ values of 0.05 or less were considered statistically significant.

\section{Results}

\section{Time Course of Tumor Progression and Detection by In Vivo Imaging}

Ex Vivo Pathology Whole mounts of excised tumoral mammary glands showed small foci of epithelial tumors as early as W4 (Fig. 1a). By W7-W8, all PyMT mice presented epithelial tumors of the mammary gland (Fig. 1b). Tumors appeared initially in the peri-areolar area and progressed throughout the mammary glands in a multifocal pattern along the ductal structures (Fig. 1a-c). Tumors extended readily and involved the whole gland by W10 (Fig. 1d).

$C T$ and $\left[{ }^{18} F\right] F D G$ Imaging $\mathrm{W} 8$ corresponded to the earliest time point at which the tumors could be detected by palpation. CT images had a low tumor-to-background ratio and did not detect mammary tumors until they reached a size sufficient to modify the body contours of the mice (W14). In contrast, mammary tumors were detected on PET images as early as W8, as shown in a series of representative $\left[{ }^{18} \mathrm{~F}\right]$ FDG-PET images acquired weekly from W8 to W16 in one mouse (Fig. 2). Mean MTV per mouse increased from $0.76 \pm 0.59 \mathrm{~cm}^{3}$ at W9 to $7.45 \pm 0.26 \mathrm{~cm}^{3}$ at $\mathrm{W} 14$, in a two-phase pattern: a slow growing phase from $\mathrm{W} 8$ to $\mathrm{W} 11$ followed by a fast growing phase thereafter. By W14, tumors had grown to large sizes and $\left[{ }^{18} \mathrm{~F}\right]$ FDG-PET showed an hypometabolic central area (see the close up at W14 in Fig. 2) that ex vivo macroscopy confirmed to be necrosis. Lung

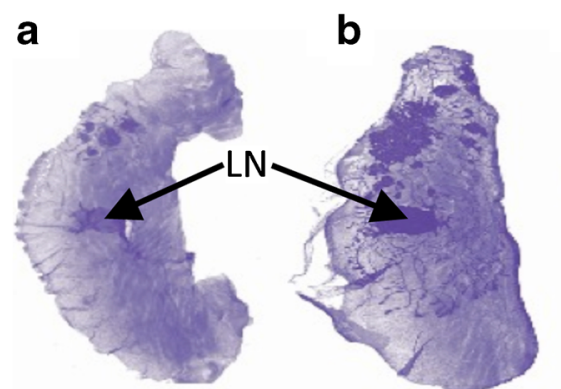

C

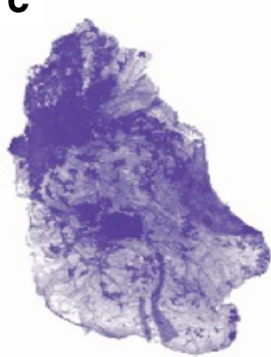

d

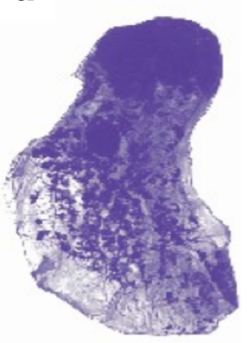

Fig. 1 Spontaneous multifocal tumoral progression. Hematoxylin-stained whole mount sample of mammary glands from untreated PyMT mice at a W4, b W7, c W9, and d W10. Arrows point to the lymph node $(L N)$ in the center of the mammary gland. 
metastasis could not be clearly identified in vivo by $\mathrm{CT}$ or PET, but a PET signal above background, possibly masking the uptake in small pulmonary lesions, was noted in the mediastinum, in addition to the myocardial uptake.

${ }^{18}$ F]FLT Imaging and Comparison with Ki-67 We found no contrast between the mammary tumors and the surrounding tissue of PyMT mice at $10 \mathrm{~min}$ (Fig. 3a) and $30 \mathrm{~min}$ (Fig. 3b) after $\left[{ }^{18} \mathrm{~F}\right] \mathrm{FLT}$ injection. Only background activity and tracer accumulation in the bladder were observed in images acquired at $30 \mathrm{~min}$ or at later times, up to $90 \mathrm{~min}$ after injection. Since $\left[{ }^{18}\right.$ F]FLT-PET is expected to reflect proliferation that is likely to be high during tumor development in the PyMT mice, we searched for the proliferation marker Ki-67 in serial histological sections of tumoral mammary glands of PyMT mice, at different stages of development.

Areas of hyperplasia, adenoma, and carcinoma were observed in mammary tumors, as previously described by Lin et al. [2]. Adenoma and carcinoma were present at W10 (Fig. 3c, d). The Ki-67 index was higher in carcinoma than in adenoma and hyperplasic areas (mean \pm SD of $44 \pm 15,34 \pm 12$, and $25 \pm 11 \%$, respectively) (Fig. 3c), and the cell density increased from W10 to W14 (Fig. 3d-f). The tumor proliferative activity assessed by the Ki-67 index was high at W14, with more than $40 \%$ positive cells (Fig. 3c). Therefore, the absence of contrast on the $\left[{ }^{18} \mathrm{~F}\right] \mathrm{FLT}$ images cannot be attributed to the absence of proliferation in mammary tumors of PyMT mice. It is noteworthy that mammary glands of normal FVB mice showed at puberty (W4-W7) a median Ki-67 index of $6 \pm 7 \%$, in agreement with ductal hyperplasia during pubertal mammary growth.
SPECT Imaging with ${ }^{99 m}$ Tc]TEC SPECT imaging with $\left.{ }^{99 m} T c\right] T E C$ showed uptake in the mammary tumors of PyMT mice as early as W6 yielding clear contrast throughout to W14 (Fig. 4a). The mean TTV increased from $0.2 \pm 0.2$ to $7.5 \pm 0.7 \mathrm{~cm}^{3}$ between $\mathrm{W} 9$ and $\mathrm{W} 14$. The tumor-to-background ratio was lower on $\left[{ }^{99 m}\right.$ Tc]TEC SPECT images than on $\left[{ }^{18} \mathrm{~F}\right]$ FDG-PET images. Staining of normal mammary glands for NIS showed an intense and ubiquitous labeling of the ducts (Fig. 4b), while in tumors, NIS was present in ductal as well as hyperplasic structures both on the plasma membrane and in the cytoplasm. NIS staining of tumoral tissue was more pronounced at W8 before treatment (Fig. 4c) than at W12 after treatment (Fig. 4d). Lung metastases were not detected on the SPECT images. To check whether this may have been the consequence of a low imaging contrast due to high background in the thoracic area (mediastinum and heart), we performed ex vivo autoradiography of lungs sampled after SPECT imaging. Interestingly, $\left[{ }^{99 \mathrm{~m}} \mathrm{Tc}\right]$ TEC uptake was found in some lung metastases but absent in other metastases (Fig. 4e, f), although at the cellular level, NIS expression was observed in all lung metastases at a level similar to that found in primary mammary tumors (Fig. 4g, h).

In Vivo Fluorescence Imaging of Blood Vessels With respect to normal mammary glands of FVB mice (Suppl. Fig. 1a), increased vascular densities and increased blood vessel diameters were clearly evident in the tumoral glands of PyMT mice injected with the vascular dye FITC-dextran and observed by FCE (Suppl. Fig. 1b). However, the parameters (mean vessel density, vessel length, and diameter) derived from the FCE images were widely variable, which precluded the use of FCE imaging as a quantitative tool for assessment of therapy efficacy in this model.

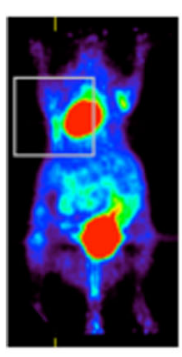

8
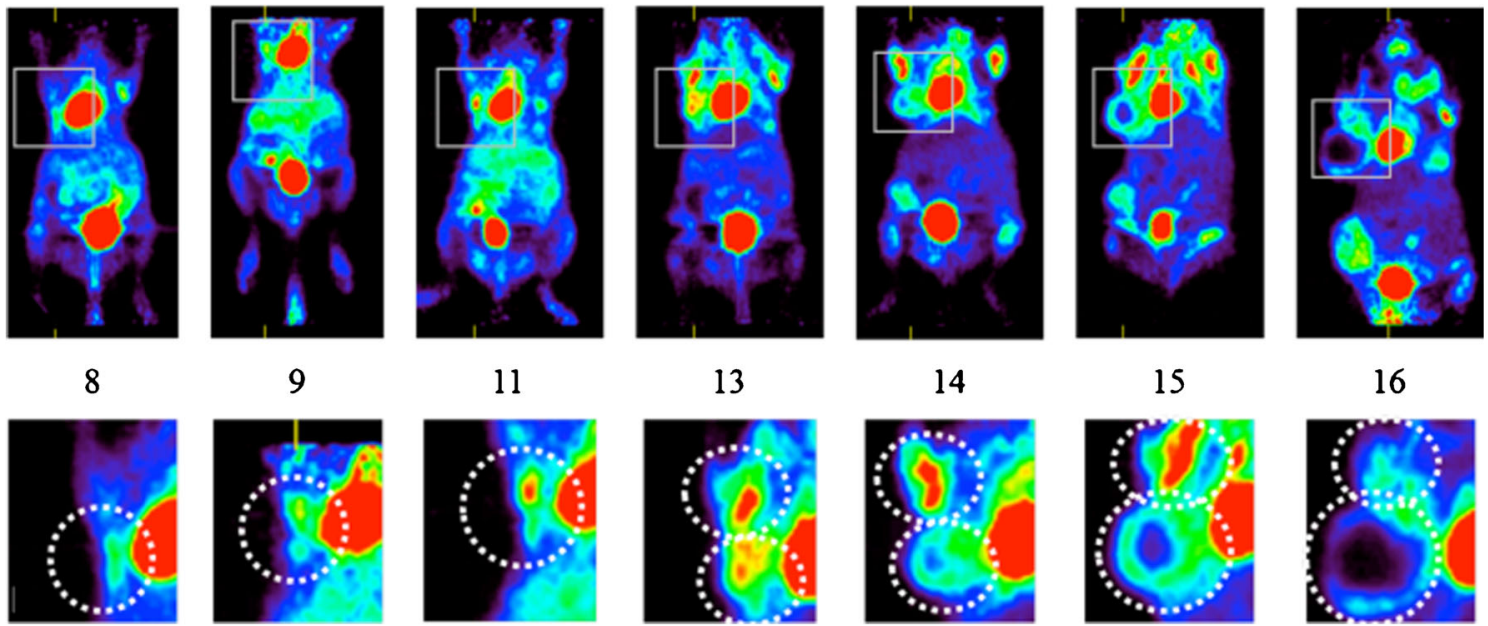

13
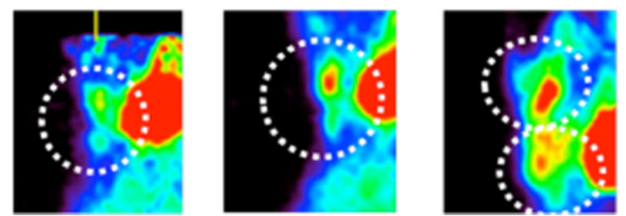

14

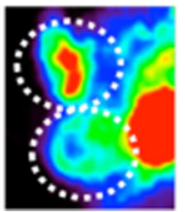

15

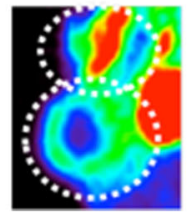

Fig. 2 Longitudinal follow-up of tumor progression from W8 to W16 with $\left[{ }^{18} \mathrm{~F}\right] \mathrm{FDG}-\mathrm{PET}$. a Whole body coronal slices of a untreated PyMT mouse and b zoomed views of the thoracic mammary tumors (numbers indicate age in weeks). Note the hypometabolic central area indicating necrosis at W14-W16. 

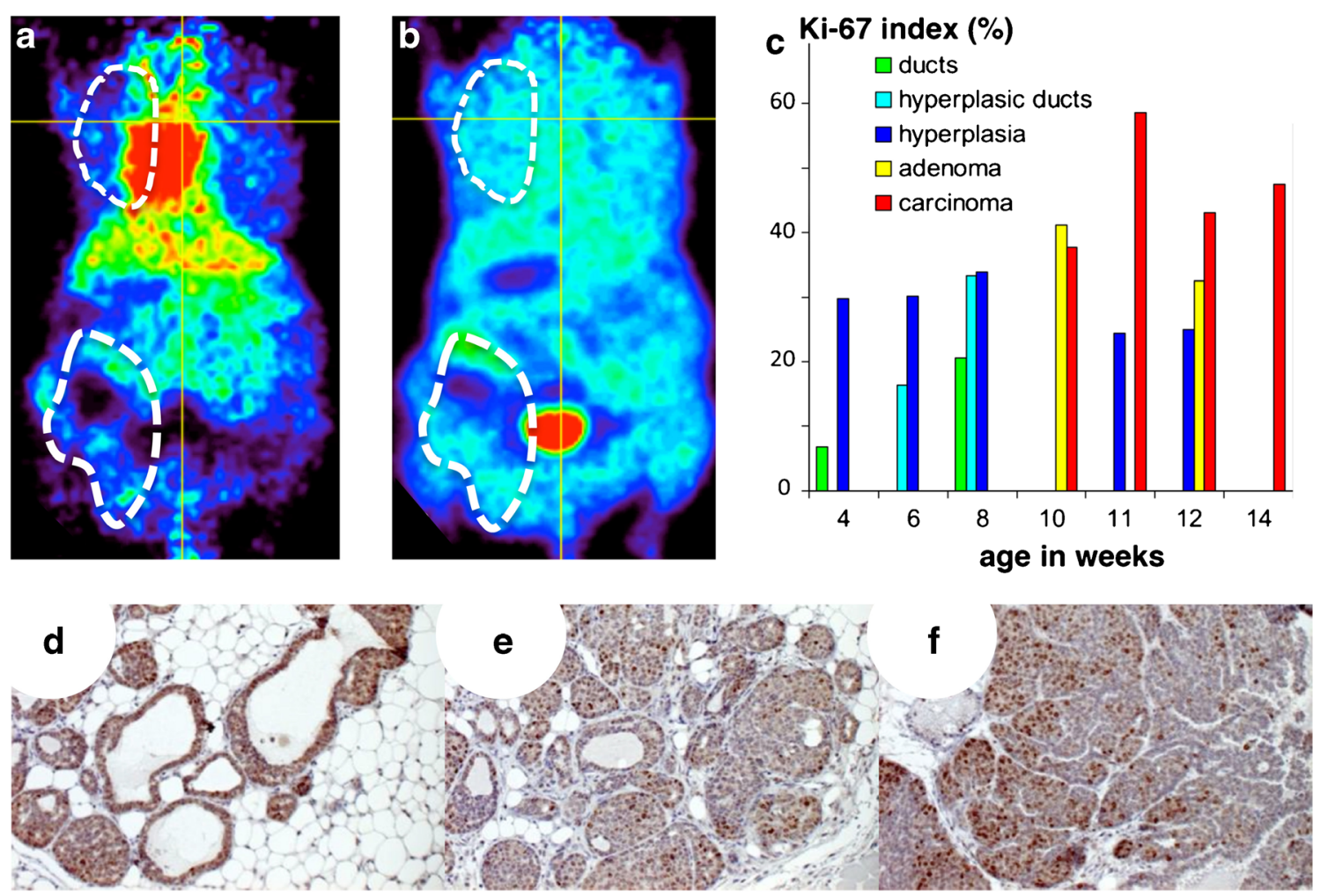

Fig. 3 Coronal PET views acquired a $10 \mathrm{~min}$ and $\mathbf{b} 30 \mathrm{~min}$ after injection of $\left[{ }^{18} \mathrm{~F}\right] \mathrm{FLT}$, showing the absence of tracer uptake in a PyMT mouse presenting large mammary tumors at W14. c Ki-67 index increases with tumor progression and is higher ( $35 \%$ or more) in carcinoma areas than in adenoma and hyperplasic areas (mean from two PyMT mice at each time point). Increase in tumor cell density and proliferation demonstrated by Ki-67 staining of mammary glands in PyMT mice at $\mathbf{d}$ W10, e W12, and $\mathbf{f}$ W14. Scale bars $200 \mu \mathrm{m}$.

\section{Assessment of the Response to Chemotherapy}

Ex Vivo Pathology and Histology Histological whole mounts of mammary glands obtained from animals treated by weekly injections of $10 \mathrm{mg} / \mathrm{kg}$ doxorubicin at $\mathrm{W} 9, \mathrm{~W} 10$, and W11 evidenced a clear regression of the lesions between W10 (Suppl. Fig. 2a) and W12 (Suppl. Fig. 2b) followed by recurrence at W14 (Suppl. Fig. 2c). In both doxorubicin- and docetaxel-treated groups, areas of tumor regression with nodular, scar, and fibrotic tissue and infiltration of inflammatory cells were observed at W10. Hyperplasic and carcinoma lesions resumed after discontinuation of chemotherapy, somewhat earlier in the docetaxel than in the doxorubicin group (W13 vs. W15). This difference might be explained by the shorter chemotherapy course for docetaxel than for doxorubicin (8vs. 14 days), as suggested by the MTV growth curve showing earlier recurrence in the docetaxel group (see below).

$\left[{ }^{18} F\right] F D G$ Chemotherapy had a major effect on MTV measured with $\left[{ }^{18} \mathrm{~F}\right] \mathrm{FDG}$ in mammary tumors (Fig. 5a). In contrast to untreated animals in which MTV increased continuously to reach maximal values at W14, MTV in animals treated from W9 to W11 by doxorubicin or docetaxel remained at pretreatment levels over the duration of chemotherapy and until 3 weeks after its discontinuation until W13-W14 (Fig. 5b). Mean MTV was $0.18 \pm 0.22$ and 0.08 $\pm 0.16 \mathrm{~cm}^{3}$ at $\mathrm{W} 9$ and $0.86 \pm 0.98$ and $1.29 \pm 1 \mathrm{~cm}^{3}$ at $\mathrm{W} 14$ in the doxorubicin- and docetaxel-treated groups, respectively (Fig. 5b). The change in MTV was statistically different between doxorubicin-treated group and untreated group $(p=0.028)$, docetaxel-treated group and untreated group $(p=0.03)$ and not between treated groups $(p=0.84)$.

After treatment discontinuation, MTV resumed its increase between W14 and time of euthanasia at W16-17, with a rate of increase in the treated group similar to the one measured in the untreated group between W11 and W14.

Tumor Growth Analysis Different tumor growth models, including a Gompertz model, were tested to fit MTV derived from $\left[{ }^{18} \mathrm{~F}\right]$ FDG-PET. Tumor growth was modeled as follows: $\mathrm{MTV}=\mathrm{MTV}_{0} \cdot e^{\mathrm{kt}}$, where $\mathrm{MTV}_{0}$ is the extrapolated volume at time 0 (i.e., at $\mathrm{W} 8$ when tumors were first observed with PET), a proportional term to equilibrate the equation dimension and was fixed to $0.03 \mathrm{~cm}^{3}$ for the analysis, $k$ is the time constant rate, $t$ is the observation time in weeks, and the growth half-life is $T_{1 / 2}=\log (2) / k$. The between-subject variability was modeled as an exponential error model and the residual variability as a combined 


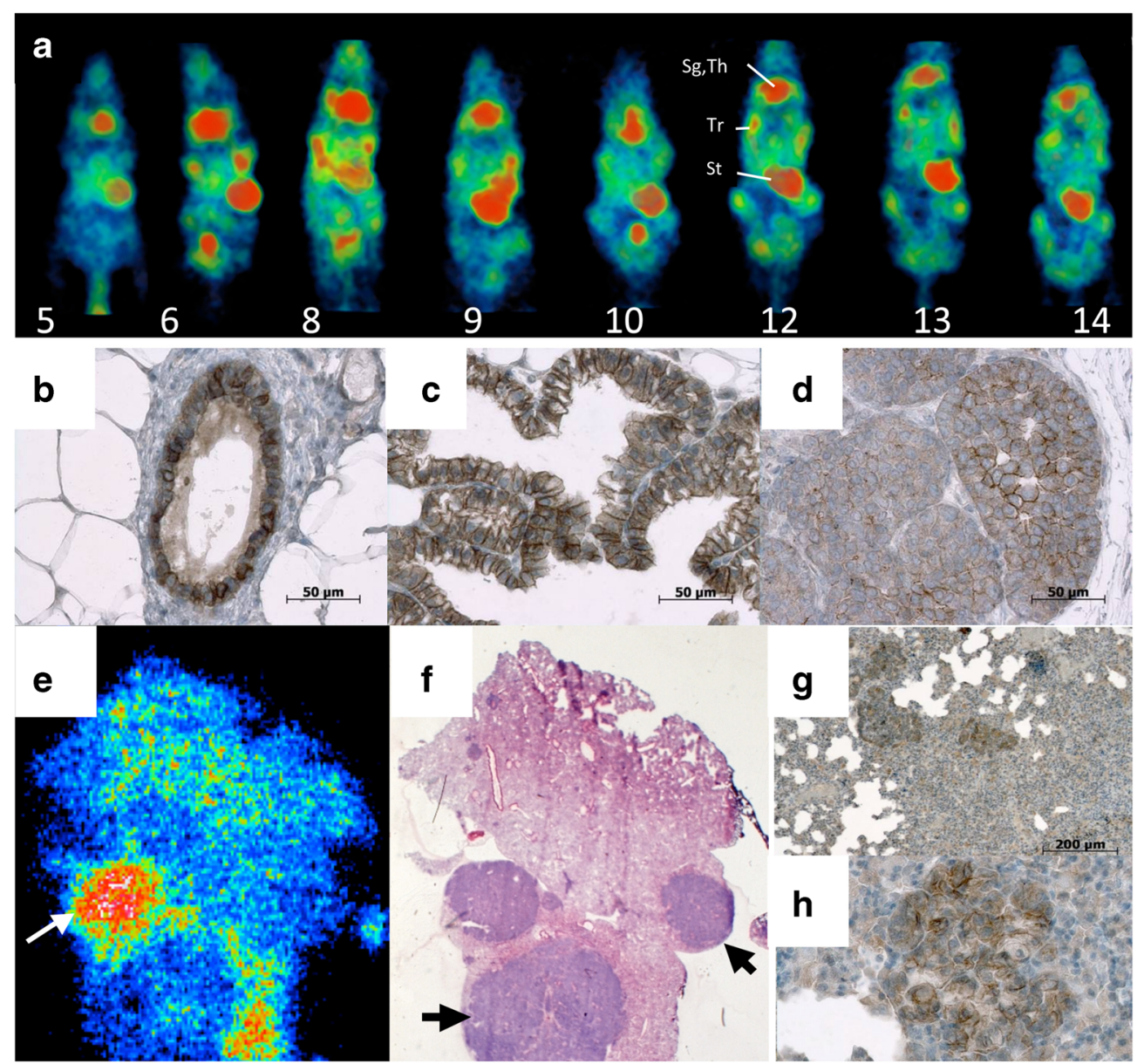

Fig. 4 a Serial SPECT images after [ $\left.{ }^{99 \mathrm{~m}} \mathrm{Tc}\right]$ TEC injection of a PyMT mouse from W5 to W14 (numbers indicate age in weeks). $\mathrm{Sg}$ salivary glands, Th thyroid gland, $\mathrm{Tr}$ mammary tumor, B/ bladder. Note $\left[{ }^{99 \mathrm{~m}} \mathrm{Tc}\right] \mathrm{TEC}$ uptake in the tumoral mammary glands already at W6. $\mathbf{b}-\mathbf{d}$ Immunohistochemistry of NIS of the mammary gland in a FVB mouse at $\mathbf{b}$ W8 and in tumoral mammary glands of PyMT mice at c W8 and d W12; scale bars $50 \mu \mathrm{m}$. e Ex vivo autoradiography of lung in a PyMT mouse at W15 after administration of $\left.{ }^{99 m} \mathrm{Tc}\right]$ TEC. $\left[{ }^{99 m} \mathrm{Tc}\right]$ TEC is present in some lung metastases (white arrow) and $\mathbf{f}$ absent in other metastases staining positively for NIS as shown with black arrows. $\mathbf{g}, \mathbf{h}$ Immunohistochemistry showing NIS labeling of the plasma membrane in lung metastases.

(proportional plus constant) error model. The $k$ estimates $(\%$ relative standard errors) were significantly different for the three groups: $0.315(4 \%), 0.145(27 \%)$, and $0.212(36 \%)$ week $^{-1}$, yielding $T_{1 / 2}$ of $2.2,4.8$, and 3.27 weeks in the untreated, doxorubicin, and docetaxel groups, respectively.

SPECT At 1 week (W10) and 2 weeks (W11) after initiation of chemotherapy with docetaxel and doxorubicin, the TTV of $\left[{ }^{99 m}\right.$ Tc]TEC uptake was unchanged or reduced with respect to pretreatment volumes. During the same time period, the TTV increased in untreated animals (Fig. 5c).

\section{Discussion}

Clinically, $70-80 \%$ of breast cancer patients respond to neoadjuvant chemotherapy, but a complete response is found in only $20-30 \%$ [13-15]. Therefore, it is important to assess response to neoadjuvant drug therapy as early as possible in order to avoid unnecessary drug exposure. Functional and metabolic properties of tissues, including tumors, respond more rapidly than gross anatomy to changes in the environment; therefore, functional and molecular imaging modalities likely to monitor tumor response to treatment more precociously than tumor size are an important field in cancer research. Because they are based essentially on the same techniques than clinical imaging, small animal imaging devices are able to provide longitudinal follow-up in preclinical studies of new anticancer drugs [16]. Here, we compared the ability of several imaging methods to document the natural course of mammary tumor development in PyMT mice. We then tested the ability of standard imaging observables derived from these methods to document precociously, precisely, and predictively the response to chemotherapy. 

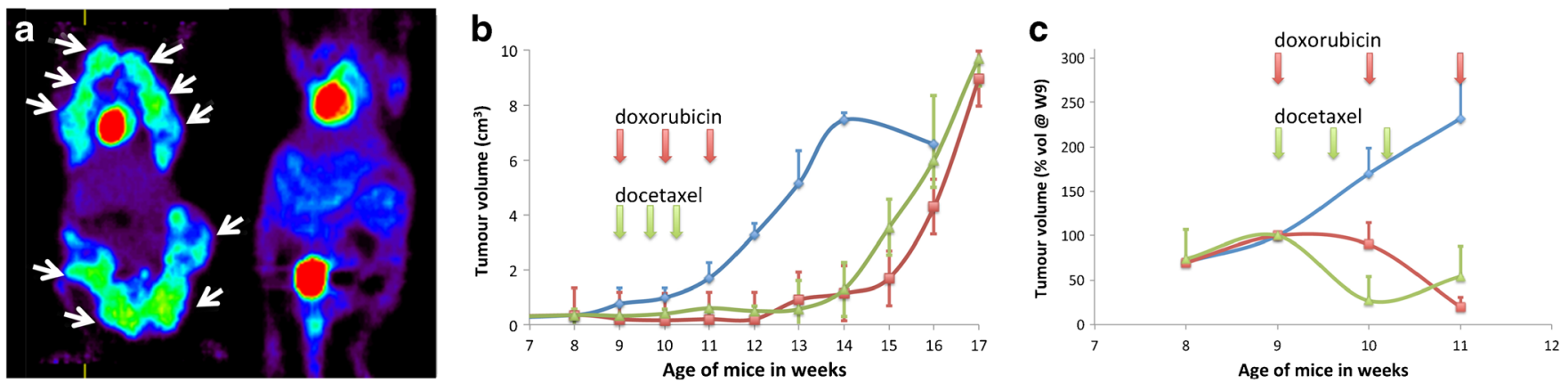

Fig. $5 \quad\left[{ }^{18} \mathrm{~F}\right] \mathrm{FDG}$-PET and $\left[{ }^{99 \mathrm{~m}} \mathrm{Tc}\right] \mathrm{TEC}-\mathrm{SPECT}$ show reduced tracer uptake with chemotherapy. a Typical $\left[{ }^{18} \mathrm{~F}\right] \mathrm{FDG}-\mathrm{PET}$ scan at W13 in an untreated (left) and a doxorubicin-treated (right) PyMT mouse. b Longitudinal assessment of the total metabolic tumor volume delineated by automatic segmentation from W8 to W14 in untreated (blue curve) and from W8 to W17 in doxorubicin- (red curve) and docetaxel-treated (green curve) PyMT mice ( $n=4$ for each group); tumor volumes are in cubic centimeter. Data points are mean \pm SD of four independent measurements. c Same for [ $\left.{ }^{99 \mathrm{~m}} \mathrm{Tc}\right] \mathrm{TEC}$ uptake on SPECT images; tumor volumes are shown as percentage of the volume at W9.

\section{How Precocious Is Detection of Cancer by In Vivo Imaging in PyMT?}

CT was able to detect the mammary tumors of the PyMT mice reliably only at W14-W15. CT is not well adapted to follow mammary carcinoma in mice because of a lower soft tissue contrast in small animals than in humans. In contrast, $\left[{ }^{99 \mathrm{~m}} \mathrm{Tc}\right]$ TEC-SPECT and $\left[{ }^{18} \mathrm{~F}\right]$ FDG-PET imaging showed a significant tumor-to-background contrast as early as W6 and W8, respectively (Fig. 4 or 2 and Suppl. Fig. 3), supporting the use of molecular imaging for early tumor detection in PyMT mice. The remarkably precocious contrast visible with $\left[{ }^{99 m} \mathrm{Tc}\right]$ TEC-SPECT at W6 could be explained by the high overexpression of NIS in premalignant hyperplasic structures. Both $\left[{ }^{99 \mathrm{~m}} \mathrm{Tc}\right] \mathrm{TEC}$ and $\left[{ }^{18} \mathrm{~F}\right] \mathrm{FDG}$ were able to follow tumor progression in the PyMT model from early stages on, even though at later stages, $\left[{ }^{99 \mathrm{~m}} \mathrm{Tc}\right] \mathrm{TEC}$ uptake as well as NIS staining tended to decrease in advanced breast cancer lesions, in contrast to $\left[{ }^{18} \mathrm{~F}\right] \mathrm{FDG}$ measurements of MTV that increased until the end of the period of observation.

\section{How Precise Is Detection of Cancer by In Vivo Imaging in PyMT?}

"Precise" imaging of cancer can mean one of two different things: (i) images that provide quantitative values of a cancer-specific molecular or functional hallmark or (ii) images that are proper surrogate markers of cancer progression.

Concerning the first aspect, in the present study, $\left[{ }^{18} \mathrm{~F}\right]$ FDG-PET and $\left[{ }^{99 \mathrm{~m}} \mathrm{Tc}\right] \mathrm{TEC}-\mathrm{SPECT}$ in PyMT mammary tumors showed increased metabolism and NIS expression, respectively. In contrast, $\left[{ }^{18}\right.$ F]FLT-PET did not reflect well the proliferation of tumor cells evidenced by Ki-67 labeling. Discrepancies between in vivo $\left[{ }^{18} \mathrm{~F}\right] \mathrm{FLT}$ uptake and proliferation have been reported in animal studies and explained by competition for $\left[{ }^{18} \mathrm{~F}\right] \mathrm{FLT}$ uptake by the high serum levels of thymidine in mice and the existence of a thymidine salvage pathway of variable importance between species and strains $[17,18]$.

Concerning the second aspect, LMA segmentation of PET dynamic images excluded the tumor regions with $\left[{ }^{18} \mathrm{~F}\right] \mathrm{FDG}$ concentrations not higher than background tissue. These regions often referred to as "necrotic" in animal studies were observed from W12 in the tumor cores of untreated mice and correspond to non-viable tumor tissue. Assessment of MTV with PET excludes these regions from the measurement of the tumor volume and improves the use of mathematical models of tumor growth and response to chemotherapy.

The size of the tumor began to increase exponentially starting at W10-11. FCE showed that this period coincides with a sudden change in the aspect of the tumor vessels and corresponds to the onset of the angiogenic switch [19], known to be associated with the progression towards carcinoma in this model [20].

Table 1. Strengths and weaknesses of the PyMT model

Strengths $(\approx$ similarities with human disease)

Orthotopic (mammary gland) and in situ (ductal) tumors Rapid: 2-3 months

Reproducible: $100 \%$ penetrance in female homozygotes

Immune competent animals

Tumor development mimics human mammary carcinogenesis stages

Expresses many markers of breast cancer including hormone receptors Lung and lymph node metastases

Weaknesses $(\approx$ difference with human disease)

Genetic, not environmental etiology

Multifocal tumors with different stages

No brain or bone metastases

Juvenile disease 


\section{How Predictive of the Response to Chemotherapy Is In Vivo Imaging in PyMT?}

$\left.{ }^{18} F\right] F D G-P E T$ The effects of doxorubicin and docetaxel were assessed by measuring the metabolic tumor volume derived from in vivo images, each animal serving as its own control, a crucial point considering the inter-individual variability of tumor growth $[5,16]$. Drugs were administered to PyMT mice at W9, 1 week after mammary tumors had become palpable. From this point of view, the present study reflects somewhat the clinical situation where imaging is challenged to report on the effect of drugs after diagnosis and before surgery.

Administration of two drugs commonly used in neoadjuvant breast cancer treatment [13-15] induced a prolonged delay of the increase in $\left[{ }^{18} \mathrm{~F}\right] \mathrm{FDG}$ uptake in the mammary tumors, at the expense of some toxicity. The ex vivo whole mount results combined with those of in vivo $\left[{ }^{18} \mathrm{~F}\right]$ FDG-PET imaging support the view that MTV assessed using $\left[{ }^{18} \mathrm{~F}\right] \mathrm{FDG}$ were related to tumor regression and not to cellular stunning after administration of cytotoxic drugs. The difference between the doxorubicin group and the docetaxel group could be related to the nature of the drug, the dosage, or the duration of administration (14 vs. 8 days for doxorubicin and docetaxel, respectively).

Overall, our results support small animal $\left[{ }^{18} \mathrm{~F}\right] \mathrm{FDG}-\mathrm{PET}$ for the assessment of response to chemotherapy, with the limitation that, as can be expected in a transgenic model in which tumorigenesis is genetically encoded, tumor progression resumes after chemotherapy discontinuation.

NIS- $\left[{ }^{99 m} T c\right] T E C$ SPECT Imaging NIS is a membrane glycoprotein of the follicular thyroid cells that actively transports iodine used for thyroid hormone biosynthesis. NIS was shown to be overexpressed in human breast cancers [21-23] and in several transgenic mice models including PyMT [21]. NIS overexpression was observed by immunohistochemistry in human breast cancers but not in normal tissue in a small series of samples [21] and confirmed in a larger series of 371 samples assessed by microarrays and histological techniques [22].

Surprisingly, in the present results, NIS overexpression was observed in normal mammary ducts in FVB mice, using three different immunohistochemical methods. This observation is not in agreement with the SPECT images showing $\left[{ }^{99 \mathrm{~m}} \mathrm{Tc}\right]$ TEC uptake only in mammary tumors and not in normal mammary glands. Although NIS expression was more heterogeneous and at a lower level in mammary tumors than in normal breast ducts (Fig. 4), the higher cellular density in tumors than in normal mammary glands induced a higher contrast with the surrounding tissue. Although lung metastases are frequent in PyMT mice, confirmed by macroscopic analysis, the absence of $\left[{ }^{99 m} \mathrm{Tc}\right]$ TEC uptake in some lung metastases on ex vivo autoradiography suggested the loss of NIS expression in metastatic lesions. Moreover, although radionuclide uptake has been shown to be correlated with NIS levels, cell surface NIS levels are variable in breast tumors [23].

The PyMT Transgenic Mouse as a Model to Study Mammary Cancer With In Vivo Imaging Carcinogenesis in the PyMT mouse presents similarities with that of human breast cancer subtype with a multistep progression from an initial hyperplasic stage to invasive carcinoma [2]. Up to a certain extent, this animal model is relevant for dynamic studies of disease evolution and therapeutic intervention and its limitations are summarized in Table 1. A main difference with human disease is the occurrence of multiple points of tumor initiation at different stages ranging from hyperplasia to carcinoma. Cancer growth occurs during puberty in PyMT mice, and at advanced stages (W10 to W16), each mouse presents several tumoral foci on each mammary gland. However, from the imaging point of view, this drawback can be limited by the use of MTV and TTV to quantify $\left[{ }^{18} \mathrm{~F}\right] \mathrm{FDG}$ and $\left[{ }^{99 \mathrm{~m}} \mathrm{Tc}\right] \mathrm{TEC}$, respectively.

\section{Conclusion}

In vivo molecular imaging of the PyMT mouse model of breast carcinoma by $\left[{ }^{18} \mathrm{~F}\right]$ FDG-PET and $\left[{ }^{99 \mathrm{~m}} \mathrm{Tc}\right] \mathrm{TEC}$ SPECT is a precise tool to allow longitudinal monitoring and predict the tumor response to chemotherapy precociously.

Acknowledgments. This study was supported by Institut National du Cancer (INCa, France) contract grant 2005-PL01 and by European Molecular Imaging Laboratories (EMIL) EU contract LSH-2004-503569.

Compliance with Ethical Standards. All institutional and national guidelines for the care and use of laboratory animals were followed.

Conflict of Interest

The authors declare that they have no conflict of interest.

Open Access This article is distributed under the terms of the Creative Commons Attribution 4.0 International License (http://creativecommons.org/licenses/by/4.0/), which permits unrestricted use, distribution, and reproduction in any medium, provided you give appropriate credit to the original author(s) and the source, provide a link to the Creative Commons license, and indicate if changes were made.

\section{References}

1. Guy CT, Cardiff RD, Muller WJ (1992) Induction of mammary tumors by expression of polyomavirus middle $\mathrm{T}$ oncogene: a transgenic mouse model for metastatic disease. Mol Cell Biol 12:954-961

2. Lin EY, Jones JG, Li P et al (2003) Progression to malignancy in the polyoma middle $\mathrm{T}$ oncoprotein mouse breast cancer model provides a reliable model for human diseases. Am J Pathol 163:2113-2126

3. Herschkowitz JI, Simin K, Weigman VJ et al (2007) Identification of conserved gene expression features between murine mammary carcinoma models and human breast tumors. Genome Biol 8:R76

4. Marangoni E, Vincent-Salomon A, Auger N et al (2007) A new model of patient tumor-derived breast cancer xenografts for preclinical assays. Clin Cancer Res 13:3989-3998

5. Dandekar M, Tseng JR, Gambhir SS (2007) Reproducibility of 18F-FDG microPET studies in mouse tumor xenografts. J Nucl Med 48:602-607 
6. Vandervoort E, Camborde ML, Jan S et al (2007) Monte Carlo modelling of singles-mode transmission data for small animal PET scanners. Phys Med Biol 52:3169-3184

7. Maroy R, Boisgard R, Comtat C et al (2008) Segmentation of rodent whole-body dynamic PET images: an unsupervised method based on voxel dynamics. IEEE Trans Med Imaging 27:342-354

8. Yun M, Oh SJ, Ha HJ et al (2003) High radiochemical yield synthesis of 3'-deoxy-3'-[18F]fluorothymidine using (5'-O-dimethoxytrityl-2'-deoxy-3'-O-nosyl-D-threopentafuranosyl)thymine and its 3-N-BOCprotected analogue as a labeling precursor. Nucl Med Biol 30:151-157

9. Oh SJ, Mosdzianowski C, Chi DY et al (2004) Fully automated synthesis system of 3'-deoxy-3'-[18F]fluorothymidine. Nucl Med Biol 31:803-809

10. Fitoussi V, Faye N, Chamming's F, et al. (2013) In vivo imaging of tumor angiogenesis using fluorescence confocal videomicroscopy. J. Vis. Exp., e50347, doi:10.3791/50347

11. Rasmussen SB, Young LJT, Smith GHP (2000) Preparing mammary gland whole mounts from mice. In: Ip MM, Asch BB (eds) Methods in mammary gland biology and breast cancer research. Kluwer Academic/ Plenum Publishing Corp, New York, pp 75-85

12. Kuhn E, Lavielle M (2005) Maximum likelihood estimation in nonlinear mixed effects models. Comput Stat Data Anal 49:1020-1038

13. van der Hage JA, van de Velde CJ, Julien JP et al (2001) Preoperative chemotherapy in primary operable breast cancer: results from the European Organization for Research and Treatment of Cancer Trial 10902. J Clin Oncol 19:4224-4237

14. Diéras V, Fumoleau P, Romieu G et al (2004) Randomized parallel study of doxorubicin plus paclitaxel and doxorubicin plus cyclophosphamide as neoadjuvant treatment of patients with breast cancer. J Clin Oncol 22:4958-4965
15. Heller W, Mazhar D, Ward R et al (2007) Neoadjuvant 5-fluorouracil, epirubicin and cyclophosphamide chemotherapy followed by docetaxel in refractory patients with locally advanced breast cancer. Oncol Rep $17: 253-259$

16. Abbey CK, Borowsky AD, McGoldrick ET et al (2004) In vivo positron-emission tomography imaging of progression and transformation in a mouse model of mammary neoplasia. Proc Natl Acad Sci U S A 101:11438-11443

17. Shields AF, Grierson JR, Dohmen BM et al (1998) Imaging proliferation in vivo with [F-18]FLT and positron emission tomography. Nat Med 4:1334-1336

18. Mankoff DA, Shields AF, Krohn KA (2005) PET imaging of cellular proliferation. Radiol Clin North Am 43:153-167

19. Folkman J (1995) Angiogenesis in cancer, vascular, rheumatoid and other disease. Nat Med 1:27-31

20. Lin EY, Li JF, Gnatovskiy L et al (2006) Macrophages regulate the angiogenic switch in a mouse model of breast cancer. Cancer Res 66:11238-11246

21. Tazebay UH, Wapnir IL, Levy O et al (2000) The mammary gland iodide transporter is expressed during lactation and in breast cancer. Nat Med 6:871-878

22. Wapnir IL, van de Rijn M, Nowels K et al (2003) Immunohistochemical profile of the sodium/iodide symporter in thyroid, breast, and other carcinomas using high density tissue microarrays and conventional sections. J Clin Endocrinol Metab 88:1880-1888

23. Dohán O, De la Vieja A, Paroder V et al (2003) The sodium/iodide symporter (NIS): characterization, regulation, and medical significance. Endocr Rev 24:48-77 